\title{
Path Analysis on Determinants of Childhood Obesity and Associated Risk Factors of Cardiovascular, Renal, and Hepatic Diseases: The CASPIAN-V Study
}

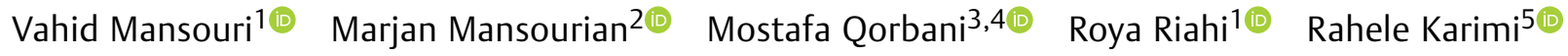 \\ Mohammad Esmaeil Motlagh ${ }^{6}$ Ramin Heshmat $^{7 \odot}$ Roya Kelishadi $^{1 \odot}$ \\ ${ }^{1}$ Department of Pediatrics, Child Growth and Development Research \\ Center, Research Institute for Primordial Prevention of Non- \\ communicable Disease, Isfahan University of Medical Sciences, \\ Isfahan, Iran \\ ${ }^{2}$ Department of Epidemiology and Biostatistics, School of Health, \\ Isfahan University of Medical Sciences, Isfahan, Iran \\ ${ }^{3}$ Department of Epidemiology, Non-communicable Diseases \\ Research Center, Alborz University of Medical Sciences, Karaj, Iran \\ ${ }^{4}$ Department of Epidemiology, Non-communicable Diseases Research \\ Center, Endocrinology, and Metabolism Population Sciences Institute, \\ Tehran University of Medical Sciences, Tehran, Iran \\ ${ }^{5}$ Department of Epidemiology and Biostatistics, School of Health, \\ Isfahan University of Medical Sciences, Isfahan, Iran \\ ${ }^{6}$ Department of Pediatrics, Ahvaz Jundishapur University of Medical \\ Sciences, Ahvaz, Iran \\ ${ }^{7}$ Department of Epidemiology, Chronic Diseases Research Center, \\ Endocrinology and Metabolism Population Sciences Institute, \\ Tehran University of Medical Sciences, Tehran, Iran \\ Address for correspondence Roya Kelishadi, MD, Department of \\ Pediatrics, Child Growth and Development Research Center, Research \\ Institute for Primordial Prevention of Non-communicable Disease, \\ Isfahan University of Medical Sciences, Hezar-Jerib Avenue, Isfahan \\ 81676-36954, Iran (e-mail: kelishadi@med.mui.ac.ir). \\ J Child Sci 2020;10:e148-e158.
}

\begin{abstract}
The interaction between several lifestyle and obesogenic environmental factors is considered as the main underlying factor for the escalating trend of childhood obesity and its adverse consequences. In this study, we assessed the mutual influence of lifestyle habits and body mass index (BMI) as well as risk factors for cardiometabolic, hepatic, and renal disorders to define the causality power of each item. This nationwide cross-sectional study was conducted as the fifth round of a school-based surveillance program. Overall, 14,800 students living in Iran were studied, and blood samples were obtained from 4,200 of them. Demographic factors, anthropometric and biochemical measures were used to define lifestyle-related latent variables as well as cardiac, renal, and hepatic risk indicators. Total, direct, and indirect effects between factors were

\section{Keywords}

- path analysis

- obesity

- lifestyle

- childhood

- adolescents analyzed using the standardized regression weights for each pathway. Data from 14,274 students (participation rate of $99 \%$ ) and 3,843 blood samples were included. All of the latent variables had a significant direct effect on $\mathrm{BMI}$, with the most potent effect of unhealthy nutrition ( $\beta \cong 0.63$ ) in boys and girls. BMI has significant direct effects on risk indicators of cardiovascular, renal, and hepatic diseases with the most powerful effect on cardiovascular risk factors $(\beta \cong-0.08)$. The most important predisposing
\end{abstract}

received

June 11, 2020

accepted

August 19, 2020
DOI https://doi.org/

10.1055/s-0040-1716917.

ISSN 2474-5871.
Copyright @ 2020 Georg Thieme Verlag License terms

KG Stuttgart · New York
(ब)(1) 
factor for obesity was unhealthy nutrition, whereas increased activity, adequate sleep, and better hygiene had protective roles. BMI shows the strongest association with indicator of cardiovascular diseases. These findings underscore the importance of implementing public health programs for the prevention of chronic noncommunicable diseases.

\section{Introduction}

The prevalence of obesity is globally emerging at an alarming rate, with a more rapid increase in developing than in developed countries. ${ }^{1-3}$ According to the World Health Organization (WHO) estimates, in 2016, there were more than 330 million overweight or obese children and adolescents aged 5 to 19 years. ${ }^{4}$

Factors that predispose children to excess weight are complex, needing a greater attempt to recognize for implementing preventative strategies. As WHO demonstrated, the interaction between several lifestyle and obesogenic environmental factors is considered as the main reason for the escalating trend of childhood obesity, as comprehensively reviewed by Sahoo et al. ${ }^{4-7}$

Moreover, childhood obesity has an essential role in predicting morbidities in adolescence and adulthood. It increases the risk of cardiometabolic disorders, as well as liver and kidney dysfunctions. ${ }^{8,9}$

The path analysis of a previous phase of this study indicated that body mass index (BMI) is the strongest determinant for cardiovascular risk factors; moreover, other variables such as unhealthy diet and low physical activity impressed their indirect effects through BMI. Many studies have evaluated the association between predisposing factors including lifestyle habits, exposure to environmental chemicals, socioeconomic status, and chronic diseases. ${ }^{10-12}$

Therefore, this study aimed to assess the interplay between lifestyle habits and BMI as well as risk factors for cardiometabolic, hepatic, and renal disorders to define the causality power of each item influencing obesity and also to determine the strength of its associations with cardiovascular, renal, and hepatic risk factors.

\section{Materials and Methods}

\section{Subjects}

This nationwide cross-sectional study was conducted in Iran as the fifth survey of a school-based surveillance program entitled Childhood and Adolescence Surveillance and Prevention of Adult Noncommunicable disease (CASPIAN)-V study. The detailed study methodology was previously published. ${ }^{13}$ In brief, the study included 14,400 students aged 7 to 18 years and 4,200 blood samples obtained from them. They were recruited by multistage random cluster sampling from urban and rural areas of 30 provinces in Iran in 2014 to 2015.

\section{Questionnaire and Measurements}

The questionnaires, which were extracted from the WHO Global School Student Health Survey, were filled out confi- dentially. These questionnaires are part of a collaborative surveillance project designed by WHO to measure and assess the behavioral risk factors and protective factors among students aged 13 to 17 years. They address 10 main areas including alcohol use, dietary behaviors, drug use, hygiene, mental health, physical activity, protective factors, sexual behaviors, tobacco use, violence, and unintentional injury. ${ }^{14}$ Their validity and reliability of Persian-translated questionnaires were confirmed previously. ${ }^{15}$ In addition to demographic characteristics, other information about many aspects of lifestyle, health behaviors, and nutrition were collected through these questionnaires. ${ }^{16}$

The age and birth date of subjects were recorded. Height, weight, and waist circumference were measured under standard protocols. BMI was calculated by dividing weight ( $\mathrm{kg}$ ) by height squared $\left(\mathrm{m}^{2}\right)$; it was categorized according to the WHO growth charts. ${ }^{17}$ Waist circumference was measured with a nonelastic tape at a point midway between the lower border of the rib cage and the iliac crest at the end of normal expiration. Systolic blood pressure (SBP) and diastolic blood pressure (DBP) were measured under the standard protocol by using mercury sphygmomanometers after 5 minutes of rest in the sitting position. ${ }^{18}$ For blood sampling, students were instructed to fast for 12 hours before the screening. A parent accompanied his or her child as blood samples were taken in the morning. After centrifugation, blood serums were transferred by the cold chain and analyzed in a central provincial laboratory that met the standards of the national reference laboratory, a WHO collaborating center in Tehran. Fasting blood sugar (FBS), triglycerides (TG), total cholesterol, low-density lipoprotein (LDL) cholesterol and high-density lipoprotein cholesterol, alanine aminotransferase (ALT), and creatinine $(\mathrm{Cr})$ were measured enzymatically by Hitachi Auto Analyzer (Tokyo, Japan).

Ethics committees of Isfahan University of Medical Sciences and other relevant national regulatory organizations approved the study (Project code: 194049). Written informed consent and oral assent were obtained from parents and students after full explanation of the study.

\section{Statistical Analysis}

We used the structural equation model (SEM), which includes confirmatory factor analysis, path analysis, partial least squares path modeling, and latent growth modeling. ${ }^{19}$ SEM is often used to assess unobservable "latent" constructs. This technique is the combination of factor analysis and multiple regression analysis, and it is used to analyze the 
Table 1 Characteristics of participants according to gender: the CASPIAN-V study

\begin{tabular}{|l|l|l|l|l|}
\hline Variables $(\boldsymbol{n}=\mathbf{1 4 , 2 7 4 )}$ & $\begin{array}{l}\text { Boys }(\boldsymbol{n}=\mathbf{7 , 2 2 8}) \\
(\text { Mean } \pm \text { SD) }\end{array}$ & $\begin{array}{l}\text { Girls }(\boldsymbol{n}=\mathbf{7 , 0 4 6 )} \\
(\text { Mean } \pm \text { SD) }\end{array}$ & $\begin{array}{l}\text { Total } \\
\text { (Mean } \pm \text { SD) }\end{array}$ & $p$-Value \\
\hline Age $(\mathrm{y})$ & $12.39 \pm 3.14$ & $12.18 \pm 3.16$ & $12.28 \pm 3.15$ & $<0.001$ \\
\hline Weight $(\mathrm{kg})$ & $42.36 \pm 18.23$ & $40.41 \pm 15.42$ & $41.39 \pm 17.11$ & $<0.001$ \\
\hline Height $(\mathrm{cm})$ & $148.15 \pm 18.77$ & $144.93 \pm 15.93$ & $146.56 \pm 17.50$ & $<0.001$ \\
\hline BMI $\left(\mathrm{kg} / \mathrm{m}^{2}\right)$ & $18.48 \pm 4.96$ & $18.53 \pm 4.43$ & $18.51 \pm 4.71$ & 0.56 \\
\hline Systolic BP $(\mathrm{mm} \mathrm{Hg})$ & $99.55 \pm 13.43$ & $98.77 \pm 12.72$ & $99.17 \pm 13.09$ & $<0.001$ \\
\hline Diastolic BP $(\mathrm{mm} \mathrm{Hg})$ & $64.08 \pm 10.70$ & $63.57 \pm 10.14$ & $63.83 \pm 10.43$ & 0.004 \\
\hline $\mathrm{TC}(\mathrm{mg} / \mathrm{dL})^{\mathrm{a}}$ & $152.96 \pm 28.06$ & $154.83 \pm 26.67$ & $153.85 \pm 27.42$ & 0.035 \\
\hline LDL-C (mg/dL) & $89.31 \pm 22.90$ & $90.86 \pm 22.26$ & $90.05 \pm 22.60$ & 0.03 \\
\hline TG (mg/dL) & $87.15 \pm 45.52$ & $89.02 \pm 44.78$ & $88.04 \pm 45.18$ & 0.021 \\
\hline FBS $(\mathrm{mg} / \mathrm{dL})^{\mathrm{a}}$ & $92.06 \pm 12.91$ & $91.20 \pm 11.14$ & $91.65 \pm 12.11$ & 0.027 \\
\hline $\mathrm{Cr}(\mathrm{mg} / \mathrm{dL})^{\mathrm{a}}$ & $0.6573 \pm 0.12$ & $0.6448 \pm 0.26$ & $0.65 \pm 0.14$ & 0.056 \\
\hline ALT $(\mathrm{U} / \mathrm{L})^{\mathrm{a}}$ & $8.49 \pm 5.11$ & $8.16 \pm 8.73$ & $8.33 \pm 7.07$ & 0.13 \\
\hline
\end{tabular}

Abbreviations: ALT, alanine aminotransferase; BMI, body mass index; BP, blood pressure; CASPIAN, Childhood and Adolescence Surveillance and Preventlon of Adult Noncommunicable disease; $\mathrm{Cr}$, creatinine; FBS, fasting blood sugar; LDL-C, low-density lipoprotein cholesterol; SD, standard deviation; TC, total cholesterol; TG, triglycerides.

${ }^{\mathrm{a}}$ The $n$ for biochemical tests was 3,843: $n$ for boys $=2,010$ and $n$ for girls $=1,833$.

structural relationship between measured variables and latent constructs.

In this model, the bootstrapping techniques in SEM with 500 bootstrap replications and factor analysis were used for each dataset to find the underlying features related to the dependent variables. Dietary habits, physical activity and sleep, environmental pollutants, stress, green space exposure, and hygiene, as well as cardiac, renal, and hepatic risk factors were the latent variables made by principal component analysis (PCA). PCA is a statistical technique that reduces the number of correlated variables by grouping indicators into one principal component which retains the utmost variance in the original variables (-Appendix 1). ${ }^{20}$ These variables were defined along with their related questions in the questionnaire presented in -Appendix 2. Original and latent variables selection was based on previous studies and WHO facts and figures on childhood obesity ${ }^{4,7}$ (-Appendix 2). Next, Pearson's correlations between variables were measured to assess the relationship between variables and to be used for structural modeling.

For all measurements, $p$-value of $<0.05$ was considered statistically significant. We used the SPSS statistical package (IBM SPSS Statistics for Windows, Version 24.0, Armonk, New York, United States: IBM Corp.) for data management and descriptive statistics, along with R 3.3.3 (R Core Team, 2017) for testing the proposed structural model.

\section{Results}

Overall, 14,274 students (participation rate of 99\%) and 3,843 blood samples (participation rate 91.5\%) were studied; they consisted of $51 \%$ boys. The participation rate for blood sampling was $91.5 \%(n=3,843)$. The details of the anthropometric and physiological characteristics of the participants are presented in -Table 1. The mean (standard deviation) BMI was 18.51 (4.71), 18.48 (4.96), and 18.53 (4.43) kg/m² in total, boys, and girls, respectively. Boys significantly had higher weight and height, but there was no difference regarding BMI in two genders. The mean level of cholesterol, LDL, and TG was significantly higher in girls. The mean level of FBS, SBP, and DBP was significantly higher in boys (-Table 1). Given our findings in the factor analysis, latent variables had rather acceptable loading factors with a mean of 0.27 on their latent variables (-Appendix $\mathbf{1}$ ).

- Table 2 presents the matrix of variance and covariance between anthropometric and biochemical variables. BMI was positively correlated with unhealthy nutrition, increased exposure to green space and sunlight, and cardiovascular, renal, and hepatic disease risk indicators. Cardiovascular disease risk indicator was positively correlated with BMI, high physical activity, cardiac and renal diseases risk indicator, and negatively with hepatic disease risk indicator. Renal disease risk indicator was positively associated with BMI and negatively with increased exposure to green space and sunlight. Hepatic disease risk indicator was positively correlated with BMI, hygienic conditions, increased exposure to environmental pollutants; it was also negatively correlated with unhealthy nutrition (-Table 2).

BMI is considered both exogenous and endogenous variables. Total, direct, and indirect effects and correlations among constructs were analyzed using the standardized regression weights for each pathway. -Fig. 1 (for boys), - Fig. 2 (for girls), and - Fig. 3 (for all of the participants) present the conceptualized models based on the measured correlation coefficients. Model goodness of fit implies that these models could successfully estimate the influence of the variables (-Appendix 3). 
Table 2 Matrix of variance and covariance between variables

\begin{tabular}{|c|c|c|c|c|c|c|c|c|c|c|}
\hline & $\mathrm{BH}$ & BMI & UN & HPA & DSS & IEG & CDI & RDI & HDI & IEP \\
\hline $\mathrm{BH}$ & 7.400 & 0.007 & 0.173 & 0.148 & -0.100 & 0.053 & -0.033 & 0.006 & 0.031 & -0.033 \\
\hline BMI & 0.009 & 20.810 & 0.059 & -0.013 & 0.008 & 0.025 & 0.106 & 0.033 & 0.021 & 0.007 \\
\hline UN & $0.173^{a}$ & $0.059^{a}$ & 5.245 & -0.036 & -0.208 & -0.010 & -0.008 & -0.012 & -0.018 & -0.17 \\
\hline HPA & $0.148^{a}$ & -0.013 & $0.036^{a}$ & 24.570 & -0.098 & 0.081 & -0.027 & 0.005 & 0.004 & 0.007 \\
\hline DSS & $-0.100^{a}$ & 0.008 & $-0.208^{a}$ & $-0.098^{a}$ & 36.740 & 0.046 & -0.002 & -0.003 & 0.001 & 0.012 \\
\hline IEG & $0.053^{a}$ & $0.025^{b}$ & -0.010 & $0.081^{a}$ & $0.046^{a}$ & 516.820 & 0.015 & -0.018 & 0.017 & -0.004 \\
\hline CDI & -0.033 & $0.106^{a}$ & -0.008 & $-0.027^{b}$ & -0.002 & 0.015 & 22.540 & 0.063 & -0.022 & -0.007 \\
\hline RDI & 0.006 & $0.033^{a}$ & -0.012 & 0.005 & -0.003 & $-0.018^{a}$ & $0.063^{a}$ & 48.690 & -0.010 & 0.002 \\
\hline $\mathrm{HDI}$ & $0.031^{b}$ & $0.021^{b}$ & $-0.018^{\mathrm{a}}$ & 0.004 & 0.001 & 0.017 & $-0.022^{\mathrm{a}}$ & -0.010 & 0.041 & -0.019 \\
\hline IEP & $-0.033^{a}$ & -0.170 & 0.171 & 0.098 & 0.067 & -0.001 & -0.033 & 1.000 & $0.006^{b}$ & 12.480 \\
\hline
\end{tabular}

Abbreviations: BH, better hygiene; CASPIAN, Childhood and Adolescence Surveillance and Preventlon of Adult Noncommunicable disease; CDI, cardiovascular diseases risk indicator; DSS, decreased social security; HDI, hepatic diseases risk indicator; HPA, high physical activity; IEG, increased exposure to green space and sunlight; IEP, increased exposure to environmental pollutants; RDI, renal diseases risk indicator; UN, unhealthy nutrition. Note: Variances are listed at the diagonal line and covariances are listed above and below the diagonal lines.

${ }^{\mathrm{a}} \mathrm{p}<0.001$.

${ }^{\mathrm{b}} \mathrm{p}<0.05$.

Structural models with path coefficients (â) are shown in - Figs. 1 to 3 , for boys, girls, and total participants, respectively. Structural models for boys and girls showed that in both genders, all of the lifestyle-related variables showed direct effects on BMI. Unhealthy nutrition, increased exposure to environmental pollutants, and increased exposure to green space and sunlight had direct positive effects on BMI in both sexes. Surprisingly, better hygiene was also

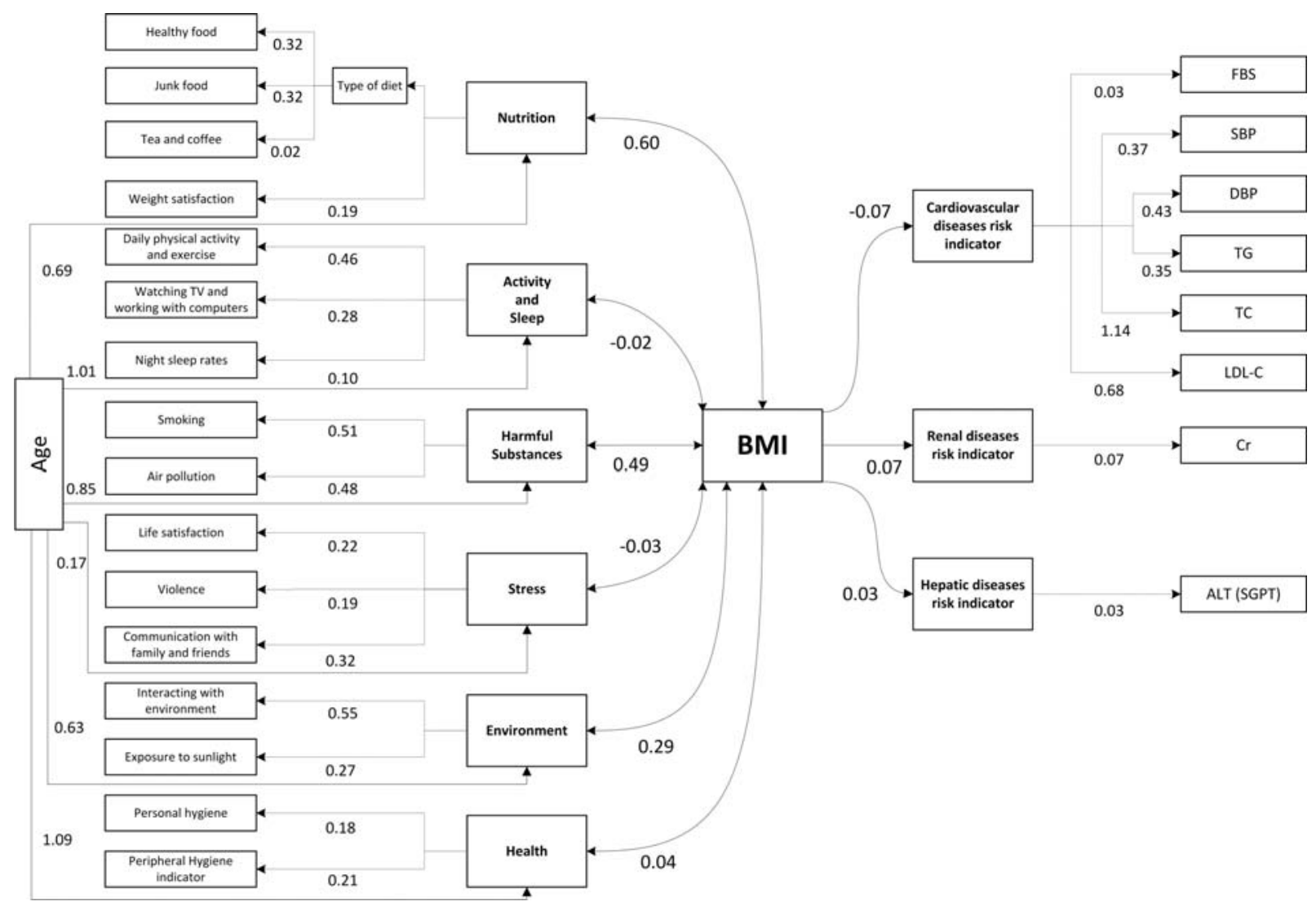

Fig. 1 Path model examining relationships among the study variables in boys: the CASPIAN-V study. ALT, alanine aminotransferase; BMI, body mass index; CASPIAN, Childhood and Adolescence Surveillance and Preventlon of Adult Noncommunicable disease; Cr, creatinine; DBP, diastolic blood pressure; FBS, fasting blood sugar; LDL-C, low-density lipoprotein cholesterol; SBP, systolic blood pressure; SCPT, serum glutamic pyruvic transaminase; TC, total cholesterol; TG, triglycerides. 


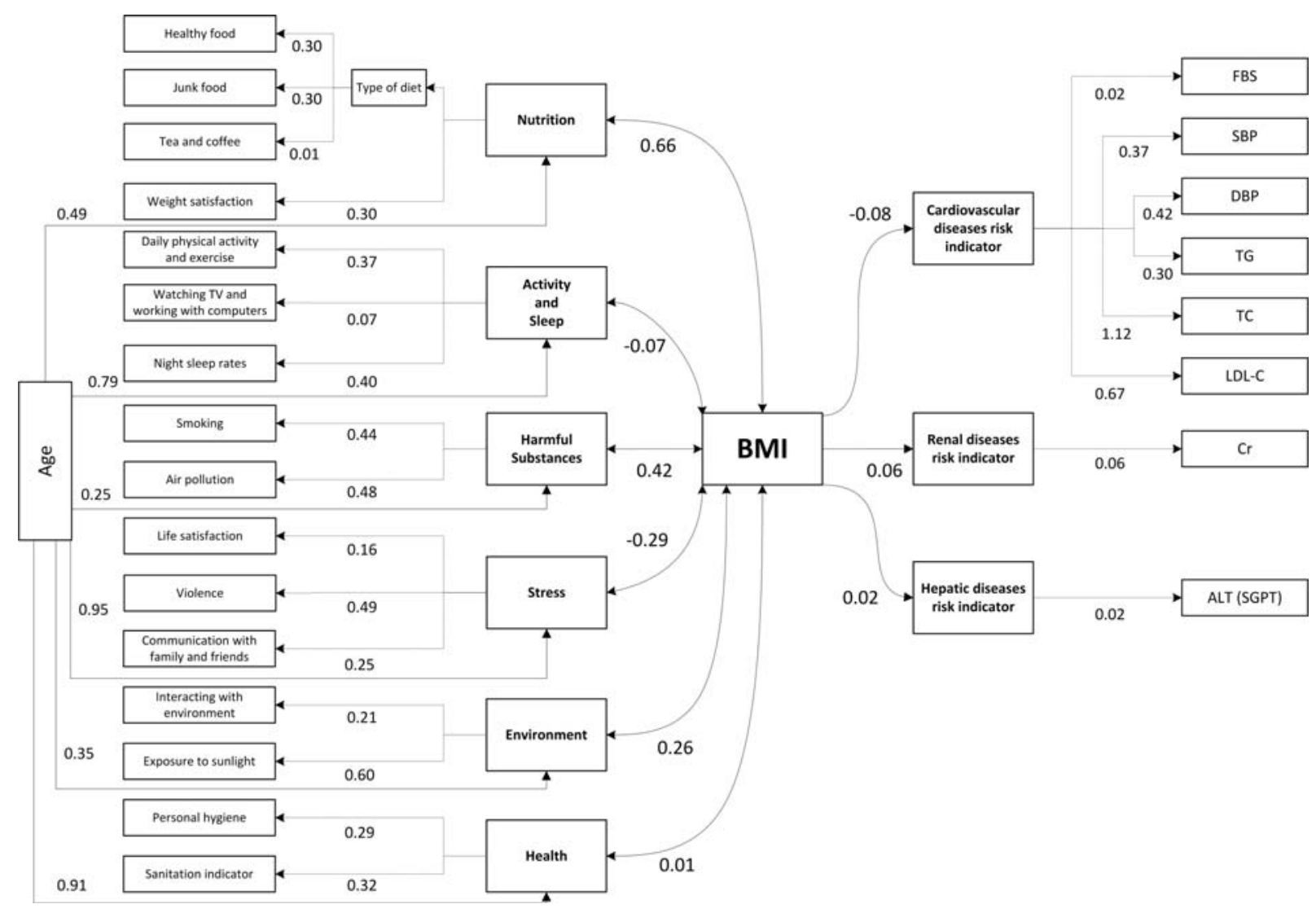

Fig. 2 Path model examining relationships among the study variables in girls: the CASPIAN-V study. ALT, alanine aminotransferase; BMI, body mass index; CASPIAN, Childhood and Adolescence Surveillance and Preventlon of Adult Noncommunicable disease; Cr, creatinine; DBP, diastolic blood pressure; FBS, fasting blood sugar; LDL-C, low-density lipoprotein cholesterol; SBP, systolic blood pressure; SGPT, serum glutamic pyruvic transaminase; TC, total cholesterol; TG, triglycerides.

positively affecting BMI in boys and girls. Higher activity, increased sleep time, decreased social security, and higher stress had a negative direct effect on BMI in both genders. In both models, the greatest positive direct effect on BMI was demonstrated by unhealthy nutrition, whereas the greatest negative $\beta$ for direct effects were applied by increased activity and higher stress in boys and girls, respectively. The path coefficients regarding the direct effects of BMI on disease risk factors showed similar patterns in boys and girls. However, higher BMI had a positive direct effect on $\mathrm{Cr}$ and ALT; surprisingly, it had negative direct effects on cardiometabolic risk factors. Although in girls the negative effect of BMI on cardiometabolic risk factors was more powerful than the positive effect on $\mathrm{Cr}$ or ALT, in boys it had the same power as $\mathrm{Cr}$ and higher than ALT (-Figs. $\mathbf{1}$ and $\mathbf{2}$ ).

Results of the structural model for all participants showed that all of the latent variables had a significant direct effect on $\mathrm{BMI}$, with the greatest effect of better hygiene in comparison to others $(\beta=-0.63, p<0.01)$. Increased physical activity and adequate sleep, increased exposure to green space and sunlight, and better hygiene had an inverse effect on BMI. No significant intervariable association was documented between latent variables. Moreover, BMI had significant positive relationships with risk indicators of cardiovascular, renal, and hepatic diseases with the strongest association with cardiovascular disease risk factors $(\beta=0.65, p<0.01)$
( - Fig. 3). The goodness of fit of models was verified using several indexes and the measured indexes are shown in -Appendix 3.

Next, to figure out the distribution of lifestyle-related variables effect through direct and indirect manners, their standardized $\beta$ of indirect effects were analyzed in total participants. As shown in - Table 3, unhealthy nutrition had a moderate positive association ( $\beta$ between 0.3 and $0.35)$ with cardiovascular and renal disease risk indicators ( $\beta$ between -0.26 and -0.4 ). In contrast, increased activity and adequate sleep and better hygiene had a moderate negative association with these risk indicators. Moreover, higher exposure to environmental pollutants had mild to moderate positive association and decreased social security and higher exposure to green spaces had lower inverse associations with these risk indicators. All of the assessed variables have mild to moderate relationship with ALT (-Table 3).

Finally, to evaluate the effect of age on this conceptualized model, we assessed the effect of age on each latent variable and their relationships. Higher ages in boys were associated with higher values regarding nutrition, sleep and activity, harmful substance use, environment, and health. Nonetheless, the relationship between BMI and disease indicators was not affected by age in both genders. In girls, higher age was associated with higher values for nutrition, sleep and 


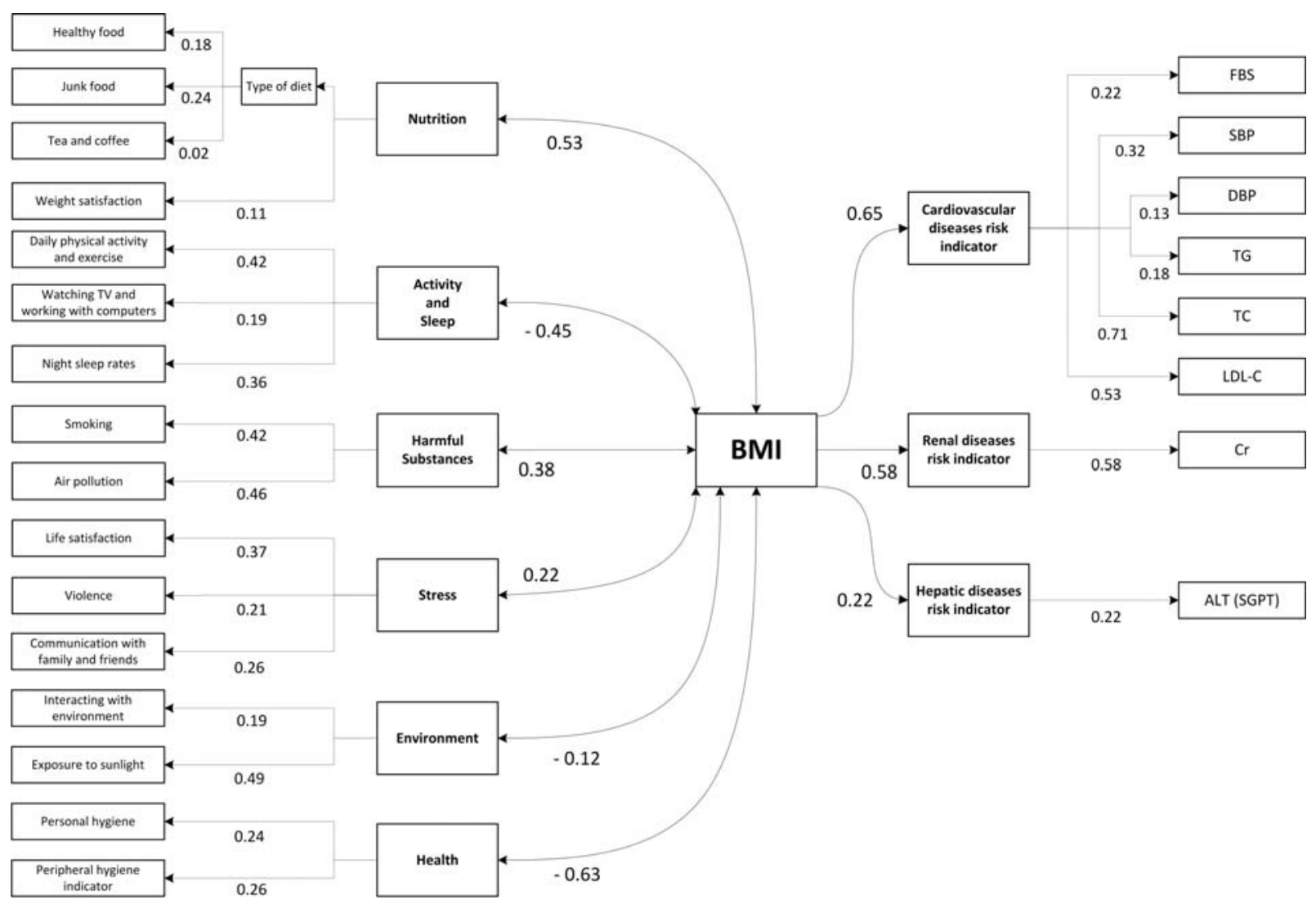

Fig. 3 Path model examining relationships among the study variables in total participants: the CASPIAN-V study. ALT, alanine aminotransferase; BMI, body mass index; CASPIAN, Childhood and Adolescence Surveillance and Preventlon of Adult Noncommunicable disease; $\mathrm{Cr}$, creatinine; DBP, diastolic blood pressure; FBS, fasting blood sugar; LDL-C, low-density lipoprotein cholesterol; SBP, systolic blood pressure; SGPT, serum glutamic pyruvic transaminase; TC, total cholesterol; TG, triglycerides.

Table 3 Standardized $\beta$ of indirect effects between different variables: the CASPIAN-V study

\begin{tabular}{|l|l|l|l|}
\hline The indirect effect of .../on ... & $\begin{array}{l}\text { Cardiovascular disease } \\
\text { risk indicator }\end{array}$ & $\begin{array}{l}\text { Renal disease } \\
\text { risk indicator }\end{array}$ & $\begin{array}{l}\text { Hepatic disease } \\
\text { risk indicator }\end{array}$ \\
\hline Unhealthy nutrition & 0.3445 & 0.3074 & 0.1166 \\
\hline Increased activity and adequate sleep & -0.2925 & -0.2610 & -0.0990 \\
\hline Increased exposure to environmental pollutants & 0.2470 & 0.2204 & 0.0836 \\
\hline Decreased social security & -0.1430 & -0.1276 & -0.0484 \\
\hline Increased exposure to green space and sunlight & -0.0780 & -0.0696 & -0.0264 \\
\hline Better hygiene & -0.4095 & -0.3654 & -0.1386 \\
\hline
\end{tabular}

Abbreviation: CASPIAN, Childhood and Adolescence Surveillance and Preventlon of Adult Noncommunicable disease.

Note: All of the indirect effects are significant.

activity, stress, and health, whereas cardiovascular, renal, and hepatic disease risk indicators were not associated with age (-Figs. 1 and $\mathbf{2}$ ).

\section{Discussion}

The main purpose of this study was to examine the indirect relationships between factors related to childhood obesity and risk factors of cardiovascular, hepatic, and renal diseases through path analysis with the mediation of their BMI. Our previous study showed that BMI is the strongest determinant for cardiovascular risk factors. Many studies have evaluated the association between contributing factors for childhood obesity with risk factors of chronic diseases, separately ${ }^{10-12}$; however, the current study provides a comprehensive model including different determinants all together.

Summarizing our findings, we found a direct positive effect of lifestyle and environmental factors on BMI except for higher activity and stress in both genders. The most powerful positive effect of contributing factors in both 
genders concerned unhealthy nutrition, whereas the most powerful negative effect was provided by higher activity. Also, there were some differences in the effects made by stress, environment, and health in each gender when compared with the total population. Assessing the effects of BMI on disease risk indicators, we found that the overall most powerful effect was on cardiovascular indices. We also found a difference in the effects of BMI and cardiovascular risk indicator in each gender compared with the total population (-Figs. 1-3).

One of the relationships investigated in this regard was the association between unhealthy nutrition and obesity. We found a strong, positive relationship between having an unhealthy diet with higher BMIs, both in boys and girls. In other words, consistent with several other studies, ${ }^{21-24}$ consuming higher amounts of fast foods and processed foods would result in higher BMI. A recent systematic review pointed out that access to fast-food restaurants was associated with overweight/obesity, ${ }^{25}$ yet the exact effect of nutritional management on obesity prevention was unclear. ${ }^{26}$ The comprehensive analysis in this study helped figure out the precise effects of nutrition among the complex interacting components. ${ }^{26}$ The importance of unhealthy nutrition prevention, as shown in this study, makes it one of the most suitable factors to be used against childhood obesity.

Another important point regarded the relationship between physical activity/exercise and adequate sleep with obesity. Although the increase in physical activity is suggested for childhood obesity since about two decades, ${ }^{27}$ there the effect of sleeping on childhood obesity is still less clear. Recent studies suggested an influential role in the duration and quality of sleep on childhood obesity. ${ }^{28-30}$ Recent systematic analysis and another population-based analysis demonstrated that a shorter duration of sleep would contribute to higher BMI. ${ }^{31,32}$ Our model revealed a moderate inverse association of increased activity and adequate sleep with BMI, either in boys and girls. This finding underscores the importance of balanced activity/sleep in childhood, which always has been on the concern of parents about overweight/obese children.

Controversial evidence exists on the effects of harmful environmental pollutants such as smoking and air pollution on obesity. ${ }^{33,34}$ A study found that mortality rate was higher in nonsmoker obese individuals than in normal weight ones, even among smokers. ${ }^{35}$ Moreover, the effects of air pollutants on obesity were reported to be different according to the demographic characteristics. ${ }^{34}$ A recent review comprehensively summarized the mechanisms underlying the association between air pollution and childhood obesity. ${ }^{36}$ We found a moderate, positive influence of harmful environmental pollutants on BMI in both genders. These findings got special importance when, according to WHO statistics, children are the main victim of secondhand smoke (in 2004, 40\% of children were exposed, globally ${ }^{37}$ ) and also, urban living increases the exposure of children to a mixture of air pollutants.

Some studies showed that lower social security increases the risk of obesity. Mental stress and its consequences such as increased appetite, lack of proper sleep quality, and decreased motivation for physical activity may play important roles in the development of obesity and failure of following weight loss behaviors. ${ }^{38-40}$ Besides, a recent study reported that neighborhood violence could flair stress up in children, eventually resulting in the same consequences. ${ }^{41}$ Our findings suggested a moderate negative relationship between stress and BMI in girls along with a weak association in boys. Because of the modulatory effects of several complex interplaying factors, considering analysis on total population, the effect of stress on BMI was positive. This could be justified based on the prominent role of body satisfaction, especially in adolescent girls along with the role of violence and mental stress in the reduction of quality of life and self-importance.

The duration of outdoor time might influence obesity in several ways, including increased sun exposure, increased activity, and on the other hand, exposure to air pollutants. Thus, the interaction of different factors would act simultaneously. The effects of outdoor exposure, which are diminishing due to urbanization and technology development, vary between different environments, cultures, and races. ${ }^{42}$ In our study, higher exposure to the outdoor environment had a positive association with BMI in both boys and girls. The complex interactions of environmental factors challenge the general acceptance of the protective role of outdoor time. Therefore, increasing the outdoor time should be first evaluated in different environments and populations, to be capable of being suggested for prevention and control of obesity.

Environmental and personal hygiene were previously documented to have a role in the development of obesity based on the gut microbiota hypothesis. ${ }^{43,44}$ This hypothesis involves childhood exposure to pathogens, which is responsible for training the immune system to become tolerant of neutral or even beneficial strain of gut microbiota. ${ }^{45}$ Several studies suggested an important role for the microbiota in the development of obesity. ${ }^{46,47}$ In our study, better hygiene had a mild positive relationship with BMI in boys and girls. However, when analyzing the whole population, a strong negative influence of hygiene was found on obesity. This difference could be due to complex interplay between genetics, altered gut microbiome, and immunity. Higher levels of hygiene would contribute to higher BMI, although the effect was small when analyzing each gender separately. However, when the total population was analyzed, due to interference of more factors including gender, genetics, or even cultural beliefs, an inverse effect of hygiene was seen. Further research is needed for introducing it as a new target for future interventions at individual and public levels.

In both genders, models showed a more pronounced relationship between BMI compared with $\mathrm{Cr}$ and ALT on cardiovascular disease risk factors. Similarly, the analysis of all the participants showed a positive relationship with cardiometabolic risk factors, as expected through metabolic syndrome.

Structural models for boys and girls showed a weak positive relationship between obesity and $\mathrm{Cr}$. Studies regarding the effect of obesity on hepatic and renal adverse events 
are rather scarce in the pediatric age group. It is shown that obesity results in intraglomerular hypertension and eventually progressing toward kidney failure. Therefore, obesity acts as one of the most influential risk factors for chronic kidney diseases. ${ }^{9}$

Nonalcoholic fatty liver disease (NAFLD) is strongly associated with obesity. However, NAFLD is almost always asymptomatic; elevated levels of liver transaminase could be detected in children. In our model, BMI was weakly associated with hepatic disease risk indicator (ALT), in both boys and girls.

\section{Study Limitations and Strengths}

One of the limitations of this study was the inability of assessing the causality effects due to the cross-sectional nature of the data. Moreover, we could not examine the pubertal status of participants.

The main strengths are the analysis of a large populationbased sample of children and a comprehensive assessment of risk behaviors and risk factors, as well as a sophisticated statistical analysis.

\section{Conclusion}

This large population-based study showed that the most important predisposing factor for obesity in both genders was unhealthy nutrition. In contrast, increased activity and adequate sleep in boys and girls, and higher stress in girls had a mild protective role against obesity. BMI was associated with indicators of cardiovascular, hepatic, and renal diseases, with the strongest association concerning cardiovascular diseases. These findings underscore the importance of implementing public health programs for the prevention of chronic noncommunicable diseases.

\section{Conflict of Interest}

None declared.

\section{Acknowledgments}

The authors are thankful to the students who participated in this study, as well as their schools' staff, executive research team, and all relevant administrators.

\section{References}

1 NCD Risk Factor Collaboration (NCD-RisC). Trends in adult bodymass index in 200 countries from 1975 to 2014: a pooled analysis of 1698 population-based measurement studies with 19.2 million participants. Lancet 2016;387(10026):1377-1396

2 Poobalan A, Aucott L. Obesity among young adults in developing countries: a systematic overview. Curr Obes Rep 2016;5(01):2-13

3 Tran BX, Dang KA, Le HT, et al. Global evolution of obesity research in children and youths: setting priorities for interventions and policies. Obes Facts 2019;12(02):137-149

4 WHO | Facts and figures on childhood obesity World Health Organization. Available at: https://www.who.int/end-childhood-obesity/facts/en/. Published 2019. Accessed July 6, 2020

5 Campbell MK. Biological, environmental, and social influences on childhood obesity. Pediatr Res 2016;79(1-2):205-211

6 Pinard CA, Yaroch AL, Hart MH, Serrano EL, McFerren MM, Estabrooks PA. Measures of the home environment related to childhood obesity: a systematic review. Public Health Nutr 2012; 15(01):97-109

7 Sahoo K, Sahoo B, Choudhury AK, Sofi NY, Kumar R, Bhadoria AS. Childhood obesity: causes and consequences. J Family Med Prim Care 2015;4(02):187-192

8 Wainwright P, Byrne CD. Bidirectional relationships and disconnects between NAFLD and features of the metabolic syndrome. Int J Mol Sci 2016;17(03):367

9 Kovesdy CP, Furth SL, Zoccali CWorld Kidney Day Steering Committee. Obesity and kidney disease: hidden consequences of the epidemic. Am J Hypertens 2017;30(03):328-336

10 Ghoorah K, Campbell P, Kent A, Maznyczka A, Kunadian V. Obesity and cardiovascular outcomes: a review. Eur Heart J Acute Cardiovasc Care 2016;5(01):77-85

11 Ajala O, Mold F, Boughton C, Cooke D, Whyte M. Childhood predictors of cardiovascular disease in adulthood. A systematic review and meta-analysis. Obes Rev 2017;18(09):1061-1070

12 Umer A, Kelley GA, Cottrell LE, Giacobbi P Jr, Innes KE, Lilly CL. Childhood obesity and adult cardiovascular disease risk factors: a systematic review with meta-analysis. BMC Public Health 2017; 17(01):683

13 Motlagh ME, Ziaodini H, Qorbani M, et al. Methodology and early findings of the fifth survey of Childhood and Adolescence Surveillance and PreventIon of Adult Noncommunicable Disease: the CASPIAN-V study. Int J Prev Med 2017;8(01):4

14 World Health Organization NCDs | Global school-based student health survey (GSHS). Available at: https://www.who.int/ncds/ surveillance/gshs/en/. Published 2018. Accessed July 6, 2020

15 Ziaei R, Dastgiri S, Soares J, et al. Reliability and validity of the Persian version of Global School-based Student Health Survey adapted for Iranian school students. Journal of Clinical Research \& Governance 2014;3(02):134-140

16 Kelishadi R, Majdzadeh R, Motlagh M-E, et al. Development and evaluation of a questionnaire for assessment of determinants of weight disorders among children and adolescents: the CASPIANIV study. Int J Prev Med 2012;3(10):699-705

17 World Health Organization WHO Multicentre Growth Reference Study Group: WHO Child Growth Standards: Length/Height-forAge, Weight-for-Age, Weight-for-Length, Weight-for-Height and Body Mass Index-for-Age: Methods and Development. Geneva: WHO; 2006

18 Flynn JT, Falkner BE. New clinical practice guideline for the management of high blood pressure in children and adolescents. Hypertension 2017;70(04):683-686

19 Kline RB. Principles and Practice of Structural Equation Modeling. New York, USA: Guilford Publications; 2015

20 Abdi H, Williams LJ. Principal component analysis. Wiley Interdiscip Rev Comput Stat 2010;2(04):433-459

21 Heerman WJ, Jackson N, Hargreaves M, et al. Clusters of healthy and unhealthy eating behaviors are associated with body mass index among adults. J Nutr Educ Behav 2017;49 (05):415-421.e1

22 Newby PK. Are dietary intakes and eating behaviors related to childhood obesity? A comprehensive review of the evidence. J Law Med Ethics 2007;35(01):35-60

$23 \mathrm{Mu}$ M, Xu LF, Hu D, Wu J, Bai MJ. Dietary patterns and overweight/ obesity: a review article. Iran J Public Health 2017;46(07): 869-876

24 Asghari G, Mirmiran P, Yuzbashian E, Azizi F. A systematic review of diet quality indices in relation to obesity. Br J Nutr 2017;117 (08):1055-1065

25 Jia P, Luo M, Li Y, Zheng JS, Xiao Q Luo J. Fast-food restaurant, unhealthy eating, and childhood obesity: a systematic review and meta-analysis. Obes Rev 2019. Doi: 10.1111/obr.12944

$26 \mathrm{Kim}$ J, Lim H. Nutritional management in childhood obesity. J Obes Metab Syndr 2019;28(04):225-235

27 Council on Sports Medicine and Fitness Council on School Health. Active healthy living: prevention of childhood obesity 
through increased physical activity. Pediatrics 2006;117(05): 1834-1842

28 Fatima Y, Doi SA, Mamun AA. Sleep quality and obesity in young subjects: a meta-analysis. Obes Rev 2016;17(11): 1154-1166

29 Felső R, Lohner S, Hollódy K, Erhardt É, Molnár D. Relationship between sleep duration and childhood obesity: systematic review including the potential underlying mechanisms. Nutr Metab Cardiovasc Dis 2017;27(09):751-761

30 Wu Y, Gong Q Zou Z, Li H, Zhang X. Short sleep duration and obesity among children: a systematic review and meta-analysis of prospective studies. Obes Res Clin Pract 2017;11(02):140-150

31 Miller MA, Kruisbrink M, Wallace J, Ji C, Cappuccio FP. Sleep duration and incidence of obesity in infants, children, and adolescents: a systematic review and meta-analysis of prospective studies. Sleep (Basel) 2018;41(04):

32 Seo SH, Shim YS. Association of sleep duration with obesity and cardiometabolic risk factors in children and adolescents: a population-based study. Sci Rep 2019;9(01):9463

33 Courtemanche $C$, Tchernis R, Ukert B. The effect of smoking on obesity: evidence from a randomized trial. J Health Econ 2018; 57:31-44

34 An R, Ji M, Yan H, Guan C. Impact of ambient air pollution on obesity: a systematic review. Int J Obes 2018;42(06):1112-1126

35 Stokes A, Preston SH. Smoking and reverse causation create an obesity paradox in cardiovascular disease. Obesity (Silver Spring) 2015;23(12):2485-2490

36 Seo MY, Kim SH, Park MJ. Air pollution and childhood obesity. Clin Exp Pediatr 2020

37 Öberg M, Jaakkola MS, Woodward A, Peruga A, Prüss-Ustün A. Worldwide burden of disease from exposure to second-hand smoke: a retrospective analysis of data from 192 countries. Lancet 2011;377(9760):139-146

38 Geiker NRW, Astrup A, Hjorth MF, Sjödin A, Pijls L, Markus CR. Does stress influence sleep patterns, food intake, weight gain, abdominal obesity and weight loss interventions and vice versa? Obes Rev 2018;19(01):81-97

39 Wilson SM, Sato AF. Stress and paediatric obesity: what we know and where to go. Stress Health 2014;30(02):91-102

40 Razzoli M, Pearson C, Crow S, Bartolomucci A. Stress, overeating, and obesity: insights from human studies and preclinical models. Neurosci Biobehav Rev 2017;76(Pt A):154-162

41 Theall KP, Shirtcliff EA, Dismukes AR, Wallace M, Drury SS. Association between neighborhood violence and biological stress in children. JAMA Pediatr 2017;171(01):53-60

42 Bento G, Dias G. The importance of outdoor play for young children's healthy development. Porto Biomed J 2017;2(05): 157-160

43 Isolauri E. Microbiota and obesity. Nestle Nutr Inst Workshop Ser 2017;88:95-105

44 Serino M, Nicolas S, Trabelsi MS, Burcelin R, Blasco-Baque V. Young microbes for adult obesity. Pediatr Obes 2017;12(04): e28-e32

45 Hunter P. The changing hypothesis of the gut. The intestinal microbiome is increasingly seen as vital to human health. EMBO Rep 2012;13(06):498-500

46 Pihl AF, Fonvig CE, Stjernholm T, Hansen T, Pedersen O, Holm JC. The role of the gut microbiota in childhood obesity. Child Obes 2016;12(04):292-299

47 Meijnikman AS, Gerdes VE, Nieuwdorp M, Herrema H. Evaluating causality of gut microbiota in obesity and diabetes in humans. Endocr Rev 2018;39(02):133-153 